\title{
Role of TMPRSS2-ERG Fusions in Prostate Cancer: Significance of Assessment in Jamaican Men \\ A Levy
}

\begin{abstract}
Prostate cancer is the leading cancer affecting men in Jamaica and is also the leading cause of male cancer-related deaths in Jamaica. This high mortality rate is primarily attributable to the eventual development of metastatic castration resistant prostate cancer which is challenging to treat. This paper discusses the pathological roles of the TMPRSS2 and ERG genes which are highly expressed in prostate cancer when compared to other cancer types. The presence of the TMPRSS2-ERG gene fusion transcript is linked to progression, metastasis and androgen regulated changes in castration resistant prostate cancer; but the status of these biomarkers have not been previously evaluated in Jamaican men with prostate cancer. The significance and implications of such an assessment is discussed herein.
\end{abstract}

Keywords: Castration resistant, prostate cancer, TMPRSS2-ERG fusion protein From: ${ }^{1}$ Pharmacology Section, Department of Basic Medical Sciences, University of the West Indies, Mona Campus, Jamaica.

Correspondence; Dr A Levy, Pharmacology Section, Department of Basic Medical Sciences The University of the West Indies, Mona Campus, Jamaica. Fax: 1 876-977-3823, e-mail: arkene_levy@yahoo.com. 


\section{Prostate cancer statistics}

Prostate cancer $(\mathrm{PCa})$ is a long latency tumor that occurs in males that are typically aged 50 years and older. Globally, more than 1.1 million cases of prostate cancer were recorded in 2012, accounting for around 8 per cent of all new cancer cases and 15 per cent in men (1). In 2015, an estimated 220,800 men will be diagnosed with PCa in the United States and an estimated 27,540 men will die from the disease making this malignancy the second leading cause of cancer-related death in men (2). In addition, African American (AA) men have the highest incidence and mortality from PCa when compared with other races (2). The Caribbean region has been recently reported as having the highest age-standardized PCa-specific mortality rate in the world at 26. 3/100 000/year (3). PCa is the leading cancer affecting men in Jamaica and is also the leading cause of male cancer-related deaths in Jamaica at 53. 9/100 000/year $(4,5)$. In other Caribbean islands such as Barbados, the incidence and mortality rates for PCa are 160. 4/100 000/year and 63. 2/100 000/year respectively (6). Trinidad and Tobago also has a high prevalence of PCa with Afro-Tobagans and Afro-Trinidadians having a threefold higher incidence than Caucasians and Indo-Trinidadians $(6,7)$. A consistent pattern of three fold higher PCa incidence amongst AfroCaribbean emigrants and their descendants in the United Kingdom when compared to the UK Caucasian population has also been reported (8). Additionally, PCa patients in the Caribbean have a worse 5- year survival rate compared to those in the US (41.6\% vs. $84.4 \%)$ (9). This evidence supports the already established genetic predisposition that drives prostate cancer pathogenesis and is worth exploring in the quest for a better understanding of prostate cancer susceptibility among Jamaicans and other Caribbean populations. 


\section{Castration resistant prostate cancer and therapeutic challenges}

In the Jamaican context, like other developing countries, there is a heightened need for better screening methods for PCa and targeted methods of ascertaining risk for this type of cancer. There is an even greater charge towards identifying new therapeutic agents that will be more efficacious at reducing the mortality rates from PCa. Mortality from this disease is usually due to resistance to Androgen Deprivation Therapy (ADT) and the eventual development of castration resistant prostate cancer (CPRC) (10). Although the disease is potentially curable by either surgical castration or chemical castration via ADT (11), approximately 33\% of patients develop resistance to these treatments with eventual increased amount of androgens, PSA, and circulating tumor cells (CTCs), leading to the more progressive and metastatic CRPC (10). The molecular etiology of the development of resistance to ADT is still not fully understood but several mechanisms including Androgen receptor (AR) amplification, gain-of-function mutation of AR, ligand-independent AR activation have been highlighted (12). Such mechanisms help to maintain AR receptor signaling in CRPC and highlights the significance of targeting AR function and signaling in this detrimental form of PCa.

\section{Role of TMPRSS2-ERG fusions in castration resistant prostate cancer}

In trying to improve survival rates, recent trends in the global management of prostate cancer have focused on the use of targeted therapies (biologics) that are directed against specific biomolecules on prostate cancer cells. Recently the two important biomarkers; ETS-related gene (ERG) and Transmembrane Protease Serine 2 (TMPRSS2) have gained recognition for their high specificity of expression in prostatic carcinomas (13). TMP RSS2 is an androgen regulated prostate-specific serine protease enzyme that is encoded in humans by the TMPRSS2 gene (13). 
ERG is a member of the erythroblastosis virus E26 (ETS) oncogene family which are transcriptional regulators that play key roles in regulating downstream target genes that are crucial for several biological processes such as cellular proliferation, differentiation, development, transformation, and apoptosis( 14).

TMPRSS2 is expressed in normal and neoplastic prostate tissue and is strongly induced by androgens in androgen-sensitive prostate cell lines (15). A major milestone in PCa research was the identification of recurrent fusions between TMPRSS2 and ERG (13). TMPRSS2-ERG is fused in PCa through deletion of genomic DNA via a homogeneous deletion site between ERG and TMPRSS2 on chromosome 21q22.2 or through translocation or both $(16,17,18)$. This rearrangement results in the formation of a TMPRSS2-ERG fusion transcript and the overexpression ERG (13). Approximately 50\% of PCa's from prostate-specific antigen (PSA) screened surgical cohorts are TMPRSS2-ERG fusion-positive, and greater than $90 \%$ of PCa's over-expressing ERG harbor TMPRSS2-ERG fusions (19). It is now established that AR regulated expression of the TMPRSS2-ERG fusion gene plays an early role in PCa development or progression and studies have confirmed that TMPRSS2-ERG fusion is a common genetic event that occurs early in prostate carcinogenesis at the transition between benign and prostatic intraepithelial neoplasia (PIN). This rearrangement has also been correlated with tumor metastasis and invasion and negative patient outcome $(20,21)$. Over eight isoforms of the TMPRSS2-ERG fusion transcript have been identified with varying levels of expression in different PCa samples (22). Evidence also suggests that PCa specimens containing the TMPRSS2-ERG rearrangement are significantly enriched for loss of the tumor suppressor gene phosphatase and tensin homologue PTEN (23), and it is already well established that aberrant 
PTEN activity is associated with poor prognosis in $\mathrm{PCa}$ (24). Further studies have confirmed that TMPRSS2-ERG rearrangement cooperates with PTEN loss to promote prostate cancer progression from high-grade prostatic intraepithelial neoplasia (PIN) to invasive adenocarcinoma $(23,25)$.

TMPRSS2 and TMPRSS2-ERG expression is decreased in response to ADT in primary PCa (26). Interestingly, the ERG levels in TMPRSS2-ERG fusion-positive CRPC are comparable with the levels in fusion gene-positive primary PC and this confirms this that TMPRSS2-ERG expression is reactivated by AR in CRPC (17). These findings prove that restored AR receptor signaling contributes to the progression to CRPC in part through the TMPRSS2-ERG axis and highlights a therapeutic platform that can be explored in the management of CRPC.

\section{Significance of assessing TMPRSS2-ERG Status in Jamaican men with PCa}

There are several studies evaluating the relationship between ethnicity and TMPRSS2-ERG expression in PCa. Galuzzi et al demonstrated that TMPRSS2-ERG gene fusion correlated with ethnicity in a multivariate analysis involving Caucasians, African-Americans, and Japanese men with PCa (18). TMPRSS2-ERG gene fusion was present in 50\% (21/42) of Caucasians, $31.3 \%$ (20/64) of African-Americans, and 15.9\% (7/44) of Japanese patients. A subsequent study found that TMPRSS2-ERG gene fusions were identified in 48/112 tumors (42.9\%) from a group of Caucasian men, while 28/105 tumors $(26.7 \%$; $\mathrm{p}=0.015)$ from African American men were positive for the gene fusion (27). Interestingly, Mosquera and colleagues recognized that the TMPRSS2-ERG fusion through deletion, which has been associated with worse prognosis, is more common in PCa of African American patients (28). 
The Jamaican and Caribbean population itself is comprised of diverse ethnicities and represents a fusion of many races including African, Caucasian, Indian and Chinese. There is no study on TMPRSS2-ERG alterations in PCa patients from Jamaica and it is therefore very important to assess for the presence and subsequent role of this gene fusion in our population where both incidence and mortality rates of $\mathrm{PCa}$ are high. A positive correlation of TMPRSS2ERG presence and PCa aggression would have significant implications towards the potential development of targeted therapies for Jamaican patients that harbor this genetic aberration. Furthermore, TMPRSS2-ERG fusion transcript can be detected in urine samples and biopsies which can lend itself to diagnostic utilization in PCa detection in Jamaica $(22,29)$.

\section{CONCLUSION}

It is very apparent that the existence of recurring gene fusions of TMPRSS2 to ERG has significant implications for understanding PCa pathogenesis and developing novel diagnostics and targeted therapeutics. Given their significant clinical implications, these lines of evidence merit TMPRSS2-ERG fusion status assessment on prostate and urine samples from Jamaican men referred for prostate biopsy on clinical suspicion of prostate cancer or undergoing radical prostatectomy on diagnosis of PCa. 


\section{REFERENCES}

1. Ferlay J, Dikshit R, Eser S, Mathers C, Rebelo M, Parkin D et al. Cancer incidence and mortality worldwide: Sources, methods and major patterns in GLOBOCAN 2012. Int J Cancer 2015; 136: E359-E386.

2. Siegel R, Miller K, Jemal A. Cancer Statistics 2015. CA: CA Cancer J Clin 2015; 65: 529.

3. Jemal A, Bray F, Ferlay J. Global Cancer Statistics. World 2011; 61: 69-90.

4. Gibson TN, Hanchard B, Waugh N. Age-specific incidence of cancer in Kingston and St Andrew, Jamaica, 2003-2007. West Indian Med J 2010; 59: 456-64.

5. Blake G, Hanchard B, Mitchell K, Simpson D, Waugh N, Wolff C, Samuels E Jamaica cancer mortality statistics, 1999. West Indian Med J 2002; 51: 64-7.

6. Hennis A, Hambleton I, Wu S, Skeete D, Nemesure B, Leske M. Prostate cancer incidence and mortality in Barbados, West Indies. Prostate Cancer 2011 Jan 27. doi:10.1155/2011/565230 [Epub 2011 Apr 17]

7. Bunker C, Patrick A, Konety B, Dhir, Brufsky A. High prevalence of screening-detected prostate cancer among Afro-Caribbeans: The Tobago prostate cancer survey high prevalence of screening-detected prostate cancer among Afro-Caribbeans: The Tobago Prostate Cancer Survey. Cancer Epidemiol Biomarkers Prev 2002; 11: 726-9.

8. Ben-Shlomo Y, Evans S, Ibrahim F, Patel B, Anson K, Chinegwundoh F et al. The risk of prostate cancer amongst black men in the United Kingdom: the PROCESS cohort study. European Urology 2008; 53: 99-105.

9. Mutetwa B, Taioli E, Attong-Rogers A, Layne P, Roach V, Ragin C. Prostate Cancer characteristics and survival in Males of African Ancestry according to place of birth: 
Data from Brooklyn - New York, Guyana, Tobago and Trinidad. Prostate 2010; 70: $1102-9$.

10. Yap T, Swanton C, de Bono1 J. Personalization of prostate cancer prevention and therapy: are clinically qualified biomarkers in the horizon? The EPMA Journal 2012; 3: 3.

11. Massard C, Fizazi K. Targeting continued androgen receptor signaling in prostate cancer. Clin Cancer Res 2011; 17: 3876-3.

12. Shen M and Cory Abate-Shen3Molecular genetics of prostate cancer: new prospects for old challenges. Genes Dev 2010; 24: 1967-2000.

13. Tomlins S, Rhodes D, Perner S, Dhanasekaran S, Mehra R, Sun X et al. Recurrent fusion of TMPRSS2 and ETS transcription factor genes in prostate cancer. Science 2005; 310: $644-8$.

14. Sreenath T, Dobi A, Srivastava S. Oncogenic activation of ERG: A predominant mechanism in prostate cancer. J Carcinogenesis 2011; 10: 37.

15. Paoloni-Giacobino A, Chen H, Peitsch M, Rossier C, Antonarakis S. Cloning of the TMPRSS2 Gene, Which Encodes a Novel Serine Protease with Transmembrane,LDLRA, and SRCR Domains and Maps to 21q22.3. Genomics 1977: 44: 309-20.

16. Perner S, Demichelis F, Beroukhim R, Schmidt F, Mosquera J, Setlur S et al. R. TMPRSS2:ERG Fusion-Associated Deletions Provide Insight into the Heterogeneity of Prostate Cancer. Cancer Res 2006; 66: 8337-41.

17. Cai C, Wang H, Xu Y, Chen S, Balk S.TMPRSS2: ERG Gene Expression in CastrationResistant Prostate Cancer. Cancer Res 2009; 69: 6027-32. 
18. Magi-Galluzzi C, Tsusuki T, Elson P, Simmerman K, LaFargue C, Esgueva R et al. TMPRSS2 -ERG Gene Fusion Prevalence and Class Are Significantly Different in Prostate Cancer of Caucasian, African-American and Japanese Patients. The Prostate 2011; 71: 489-97.

19. Tomlins S, Laxman B, Varambally S, Cao X, Yu J. Role of the TMPRSS2-ERG Gene Fusion in Prostate Cancer. Neoplasia 2008; 10:177-88.

20. Demichelis F, Fall K, Perner S, Andrén O, Schmidt F, Setlur S, et al. TMPRSS2: ERG gene fusion associated with lethal prostate cancer in a watchful waiting cohort. Oncogene 2007; 26: 4596-9.

21. Klezovitch O, Risk M, Coleman I, Lucas J, Null M, True L et al. A causal role for ERG in neoplastic transformation of prostate epithelium. Proc Natl Acad Sci USA. 2008; 105: $2105-10$.

22. Nguyen P, Violette P, Chan S, Tanguay S, Kassouf W, Aprikian A et al. A Panel of TMPRSS2: ERG Fusion Transcript Markers for Urine-Based Prostate Cancer Detection with High Specificity and Sensitivity. Eur Urol. 2011; 59: 407-14.

23. Carver B, Tran J, Gopalan A, Chen Z, Shaikh S, Carracedo A et al. Aberrant ERG expression cooperates with loss of PTEN to promote cancer progression in the prostate Nat Genet 2009; 41: 619-24.

24. Squire J. TMPRSS2-ERG and PTEN loss in prostate cancer. Nat Genet 2009; 41: 50910.

25. King J, Xu J, Wongvipat J, Hieronymus H, Carver B, Leung D et al. Cooperativity of TMPRSS2-ERG with PI3-kinase pathway activation in prostate oncogenesis. Nat Genet $2009 ; 41:$ 524-6. 
26. Mostaghel E, Page S, Lin D, Fazli L, Coleman I, True L et al. Intraprostatic androgens and androgen-regulated gene expression persist after testosterone suppression: therapeutic implications for castration-resistant prostate cancer. Cancer Res 2007; 67: 5033-41.

27. Khani F, Mosquera J, Park K; Srivastava A, Tewari A, Mark R et al. Differences in TMPRSS2-ERG Gene Fusion and SPINK1 Overexpression in Prostate Cancer in African-American and Caucasian Men. J Urology 2012; 187: e132

28. Mosquera J, Mehra R, Regan M, Perner S, Genega E, Bueti G et al. Prevalence of TMPRSS2-ERG Fusion Prostate Cancer among Men Undergoing Prostate Biopsy in the United States Clin Cancer Res 2009; 15: 4706-11

29. Laxman B, Tomlins S, Mehra R, Morris D, Wang L, Helgeson B et al. Noninvasive Detection of TMPRSS2:ERG Fusion Transcripts in the Urine of Men with Prostate Cancer. Neoplasia 2006; 8: 885-8. 